\title{
Numerical and Experimental Demonstration of Inverse Designed Low-index Polarization-insensitive Wavelength Demultiplexer
}

Abdullah Icli ( $\square$ iclibera@gmail.com )

Department of Guidance and Control, Roketsan Missiles Inc.

Ahmet Alpkilic

TOBB University of Economics and Technology

Yusuf Yilmaz

TOBB University of Economics and Technology

Bumin Yildirim

Korea Advanced Institute of Science and Technology

Mirbek Turduev

TED University

Hamza Kurt

Korea Advanced Institute of Science and Technology

\section{Research Article}

Keywords: computational inverse design, polarization-insensitive wavelength division multiplexer (PIWDM), TM polarization

Posted Date: January 5th, 2021

DOl: https://doi.org/10.21203/rs.3.rs-137530/v1

License: (c) (i) This work is licensed under a Creative Commons Attribution 4.0 International License.

Read Full License 


\title{
Numerical and Experimental Demonstration of Inverse Designed Low-Index Polarization-Insensitive Wavelength Demultiplexer
}

\author{
Abdullah B. Icli ${ }^{1,2, *}$, Ahmet M. Alpkilic ${ }^{2}$, Yusuf A. Yilmaz ${ }^{2}$, Bumin K. Yildirim ${ }^{3,4}$, Mirbek \\ Turduev $^{4}$, and Hamza Kurt ${ }^{2,3}$ \\ ${ }^{1}$ Department of Guidance and Control, Roketsan Missiles Inc. \\ ${ }^{2}$ Department of Electrical and Electronics Engineering, TOBB University of Economics and Technology, Sogutozu, \\ Sogutozu Cd. No:43, 06510 Cankaya/Ankara \\ ${ }^{3}$ School of Electrical Engineering, Korea Advanced Institute of Science and Technology (KAIST), Daejeon, 34141, \\ Republic of Korea \\ ${ }^{4}$ Department of Electrical and Electronics Engineering, TED University, On Cebeci, Ziya Gokalp Cd. 48/A, 06420 \\ Cankaya/Ankara \\ *iclibera@gmail.com
}

\begin{abstract}
The computational inverse design has paved the way for the design of highly efficient, compact, and novel nanophotonic structures beyond human intuition and trial-and-error approaches. Consequently, with this new design power, the exploration and implementation of multi-objective, complex, and functional nanophotonic devices become feasible. Herein, we used a recently emerged inverse design framework to demonstrate the design of a $1 \times 2$ polarization-insensitive wavelength division multiplexer (PIWDM) made of a low-refractive-index material with an index of 1.55. The designed PIWDM structure successfully steers toward the targeted channels for $1.30 \mu \mathrm{m}$ and $1.55 \mu \mathrm{m}$ with TE and TM polarizations, respectively. The transmission values were -2.42 and $-2.18 \mathrm{~dB}$ for TE and -2.19 and $-2.23 \mathrm{~dB}$ for TM polarization at the upper and lower waveguides, respectively. Taking advantage of the design with a low refractive index material, we scaled the structural dimensions corresponding to the microwave region, fabricated the compact device using a 3D printer, and conducted an experiment as a proof of concept. The experimentally verified PIWDM structure shows a power transmission efficiency of over $-2.42 \mathrm{~dB}$ and a crosstalk value of less than $-11.45 \mathrm{~dB}$ for the targeted wavelengths.
\end{abstract}

\section{Introduction}

During the last two decades, researchers have tried to merge nanophotonics applications with optical communication technologies to design reliable, compact, and multifunctional devices. Because photonic integrated circuits require less operating power and support relatively inexpensive, robust, and efficient optical communication systems, any modern design must comply with these criteria ${ }^{1}$. Moreover, the integration of fully dielectric nanophotonic components such as multiplexing couplers, waveguides, lasers, and light-emitting diodes into a single device can lead to the miniaturization of optical circuits with high data processing capabilities such as silicon chips used for integrated electronics or optical communication technologies. Frequently encountered optical interconnection devices in optical communication systems include steering devices such as power splitters, mode division demultiplexers, and specifically wavelength demultiplexers (WDMs) ${ }^{2,3}$. Owing to the use of WDMs, differently modulated optical signals can be conveyed by different wavelengths. To develop a WDM, two different approaches are available in the literature as passive and active demultiplexing, the first of which is based on prisms, diffraction gratings, and spectral (frequency) filtering devices, and the latter is based on a combination of passive components and tunable detectors, where each detector is tuned to a specific frequency ${ }^{4}$. Here, the preferable method for designing integrated WDMs is using a passive demultiplexing approach, which is feasible in terms of cost and power efficiency. In this regard, the design of passive and low-loss demultiplexing devices with compactness and multifunctional characteristics is a challenging issue, and its solution is widely demanding. By contrast, conveying different wavelengths to specific waveguides can be considered a great opportunity to redesign new compact and efficient optical communication systems. In this direction, in recent decades, WDMs have attracted significant attention with state-of-the-art nanophotonic design tools and approaches ${ }^{5-14}$. Optically conveying a light source with minimum loss is crucial in the design of efficient and integrable WDMs. Here, the important parameter of light guiding structures is their sensitivity to optical scattering losses resulting from input/output surfaces. In this regard, the application of 
low-index-contrast (LIC) optical structures/waveguides made by polymers can be considered an effective solution for efficiently guiding a light source ${ }^{15-18}$. Moreover, LIC optical structures have low loss and can achieve a misalignment-tolerance coupling with other optical structures. In addition, these structures facilitate massive photonic interconnects and packaging. For these reasons, in this study, we preferred to work with a material with a low refractive index in the design of the WDM structure. In general, two types of design approaches for nanophotonic devices have been shown in the literature. The first method is a conventional educated intuition-based approach. With this method, the designers need to practice their skills regarding a parameter search and analytical formulation or incorporate search algorithms to reach the targeted device ${ }^{5-10,19-29}$. By contrast, the second approach is based on advanced algorithms and combined simulations to seek a solution that minimizes (or maximizes) a single objective or multi-objective related to the desired nanophotonics functionalities, also known as an "Inverse Design." Because non-heuristic methods provide an effective optimization of all structural parameters that are impossible with conventional methods, they have been used effectively in the design of nanophotonic devices ${ }^{11-14,19-31}$. One example of the first method is integrating a Mach-Zehnder interferometer (MZIs) and $S i_{3} N_{4}$ on a silicon platform for the design of an on-chip polarization-insensitive WDM (PIWDM) device with a footprint of only $2.5 \mathrm{~mm} \times 0.9 \mathrm{~mm}^{10}$. The device was able to split four different wavelengths using conventional design methods at quite wider footprints. There are many building blocks in a typical design, so that it is impossible to simulate even a small number of the structures made from these blocks. This makes the intuitive approach hard to proceed for the designer ${ }^{24}$. By contrast, to achieve the optimum structure by using inverse design techniques, the gradient-based inverse design technique called "objective-first" is exploited. This approach is used in different nanophotonic applications such as WDMs ${ }^{12}$, optical diodes ${ }^{28}$, and metalenses with a tunable focal distance ${ }^{29}$. As the main limitation in the design of photonic structures, the structure to be fabricated can only be made using a limited number of different materials to realize the variation in the permittivity distributions through the structure. Therefore, structures with a smaller number of different materials are preferable for manufacturing in terms of cost, fabrication feasibility, and material availability. In this context, the final design must satisfy fewer differences in the permittivity distribution, vertical homogeneity as required by lithography, and targeted sizing constraints. In objective-first inverse design method, the final optimized structure has a continuous dielectric distribution, which is obtained by the gradient of the target function during the optimization process. Because it is impossible to fabricate a structure with an arbitrarily distributed dielectric constant, the optimized structure should be discretized to a producible single-material platform. The main problem in the discretization of a binary structure leads to discrepancies in the optical efficiency of the optimized structure. To overcome this issue, we used Stanford Photonic INverse design Software (SPINS-b) tool for inverse design, which is available as an open-source package $^{24}$. This study presents the design and analysis of a three-dimensional (3D) WDM device, which operates at both transverse-magnetic (TM) and transverse-electric (TE) polarizations and is realized using inverse design method. Because of the different interaction nature of the TE and TM polarized light with the designed metastructure, the combination of connected and isolated complex-shaped irregular gratings offers a smart design approach to independently manipulate the polarization state of the on-chip light propagation. For wavelength demultiplexing, we aim to split two specific wavelengths $(1.30 \mu \mathrm{m}$ and $1.55 \mu \mathrm{m}$ ) into different optical channels. To experimentally verify the designed structure, we scaled the structure up to adapt it to the microwave region and fabricated it using fused deposition modeling (FDM) with a polylactide (PLA) thermoplastic polyester material. Finally, we experimentally demonstrated its polarization-insensitive wavelength separation capability.

\section{Methods and Simulations}

In this study, we used SPINS-b computational gradient-based optimization framework to design a PIWDM structure. SPINS-b aims to produce a fabricable nanophotonic device layout by receiving a user-defined objective function (performance criteria) as the input design parameters. SPINS-b does not start with computations, but it first produces an image of the problem by setting up a "problem graph." The problem graph is made up of so-called nodes, which are the building blocks of the problem. These nodes are connected to each other in such a way that the product of a function feeds another function. To compute the final node-objective function input by the designer, for the problem graph, SPINS-b starts a series of transformations of an initial randomly generated structure that is surrounded by the design area defined by the designer. These transformations contain continuous and discrete optimizations and a final discretization process. Although continuous and discrete optimization stages are subsequently implemented, the discretization process at the end forces fabrication constraints for the device. To achieve a final device, SPINS-b applies iterative convergence to reach the objective. Each iteration is a transformation, and each transformation calculates the problem graph. To compute the nodes in the problem graph for each transformation, SPINS-b uses its own implementation of the finite-difference frequency-domain (FDFD) method for the optimization of the light matter interaction of the optical device. In short, the optimization method is combined with the FDFD method to achieve an optimization task defined by an objective function. Here, the objective function is an optimization problem defined by the designer. In our case, polarization-insensitive wavelength demultiplexing is defined as an optimization problem and is defined analytically as follows: 


$$
\min _{\varepsilon, E_{i}, H_{i}} \sum_{A \in E, H} f\left(\left|c_{1} A_{1}\right|^{2}, 1\right)+f\left(\left|c_{2} A_{2}\right|^{2}, 1\right)
$$

subject to

$$
\begin{aligned}
& \nabla \times \varepsilon^{-1} \nabla \times H_{i}-\mu_{0} \omega_{i}^{2} H_{i}=\nabla \times \varepsilon^{-1} J_{i} \\
& \nabla \times \mu^{-1} \nabla \times E_{i}-\omega_{i}^{2} \varepsilon E_{i}=-i \omega_{i} J_{i} \\
& \text { for } i=1,2
\end{aligned}
$$

where $\omega_{i}$ represent the frequencies at $1.30 \mu \mathrm{m}(\mathrm{i}=1)$ and $1.55 \mu \mathrm{m}(\mathrm{i}=2) ; E_{i}$ and $H_{i}$ are the electric and magnetic fields for the frequency $\omega_{i} ; J_{i}$ is the excitation current density at $\omega_{i}$; A represents the $\mathrm{E}$ and $\mathrm{H}$ fields; $c_{i}$ is the overlap vector for $A_{i}$; the top $(i=1)$ and bottom $(i=2)$ output waveguide such that $\left|c_{1} A_{1}\right|^{2}$ and $\left|c_{2} A_{2}\right|^{2}$ are the power intensities forming the fundamental mode of the upper and lower waveguide, respectively; and $f(x, y)=(x-y)^{2}$. Here, the main objective is to efficiently transfer optical power to the target channel regardless of the polarization. The objective function is an equally weighted sum of four sub-objectives, which corresponds to maximizing the transmission through the top waveguide at $1.30 \mu \mathrm{m}$ and the transmission through the bottom waveguide at $1.55 \mu \mathrm{m}$ for both polarizations. In order not to force the objective function to become more complex, terms for reducing the crosstalk are not added to the objective function. Thus, the reduction in crosstalk was provided indirectly. It is important to note that during the optimization process, each iteration was analyzed using 2D FDFD method, where the perfectly matched layer (PML) is used as the boundary condition. The optimization process was terminated at the 1200th iteration, and the final optimized structure was analyzed using 3D finite-difference time-domain (FDTD) method ${ }^{32}$.

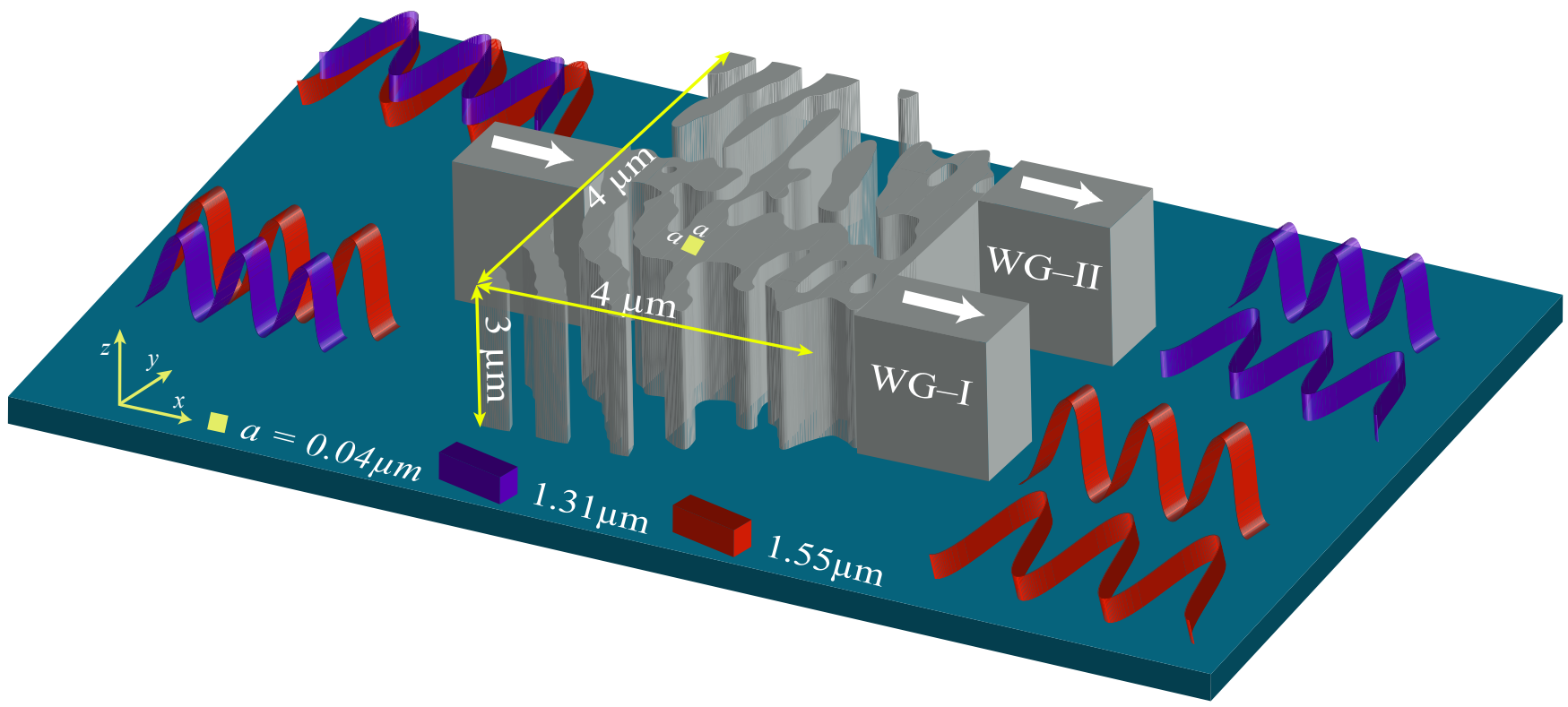

Figure 1. Schematic depiction of the PIWDM device. Incident light with two different polarizations (vertical and horizontal) oscillating at two specific wavelengths (two colors) is separated irrespective of its polarization.

As stated before, in this study, we focus on the polarization-insensitive wavelength demultiplexing effect. Fig. 1 presents a visual representation of the demultiplexing schematic of the designed PIWDM where the PIWDM splits the incident broadband light $(1.2-1.6 \mu \mathrm{m}$ ) into guided waves of two specified target wavelengths of $1.30 \mu \mathrm{m}$ (channelized to the upper waveguide (labeled WG-II)) and $1.55 \mu \mathrm{m}$ (channelized to the lower waveguide (labeled WG-I)) for both TE and TM polarizations. The output waveguides in the structure are located laterally to the input waveguide channel. The lattice constant parameter "a" defines the physical dimension of the unit cell and is fixed at $0.04 \mu \mathrm{m}$. The waveguide widths of the structure were selected as $0.80 \mu \mathrm{m}$ (20a) and the input waveguide was positioned in the middle of the design area of $4 \mu \mathrm{m} \times 4 \mu \mathrm{m} \times 3 \mu \mathrm{m}(100 \mathrm{a} \times 100 \mathrm{a}$ $\times 80 \mathrm{a})$ in the $\mathrm{x}-, \mathrm{y}-$, and $\mathrm{z}$-directions, respectively. The centers of the output waveguides are located $1 \mu \mathrm{m}$ above and below the middle of the design area. PIWDM is designed for a light source with fundamental TE and TM polarizations with non-zero components of $H_{z}$ and $E_{z}$ in the z-direction, respectively. The computational domain is surrounded by perfectly matched 
layers (PML) to eliminate possible back-reflections ${ }^{33}$. Because we intended to verify the designed structure experimentally in the microwave region, the design material is PLA material with a low relative permittivity of $2.4025^{34}$ To validate the functional operation, 3D FDTD simulations were conducted for TE and TM polarized light, where numerical calculations were conducted only for the magnetic $H_{z}$ and electric $E_{z}$ fields, respectively. Fig. 2 shows the operation of the designed PIWDM, where the demultiplexer device wavelengths of $1.30 \mu \mathrm{m}$ and $1.55 \mu \mathrm{m}$ into WG-II and WG-I, respectively, for both TE and TM polarizations. In addition, in the same figure, to provide the efficiency performance of the PIWDM structure, we calculated the transmission efficiency plots of the regarded channels within the operating wavelength range of $1.2-1.6 \mu \mathrm{m}$. It is important to note that the input channel is excited by TE and TM polarized mode source.

a)

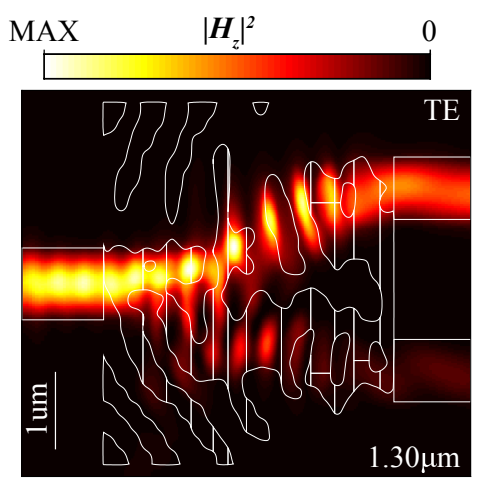

d)

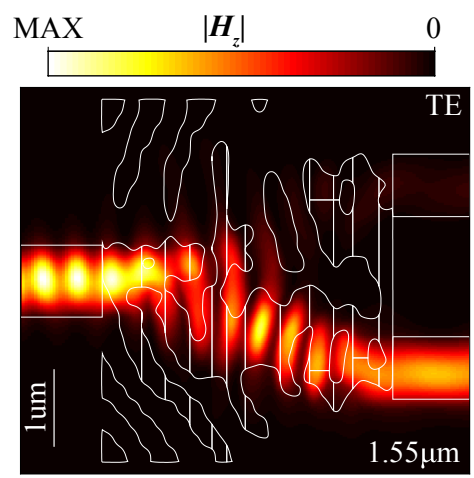

b)

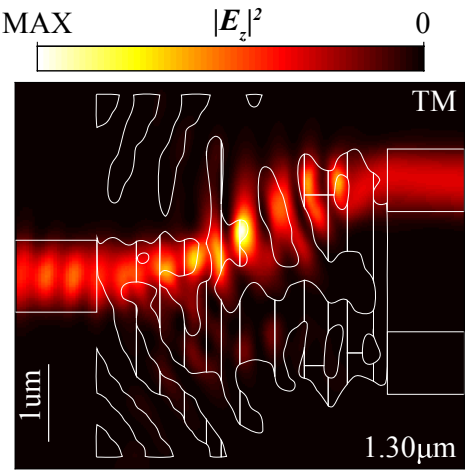

e)

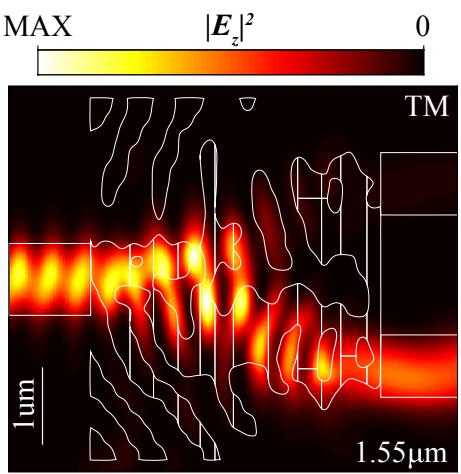

c)

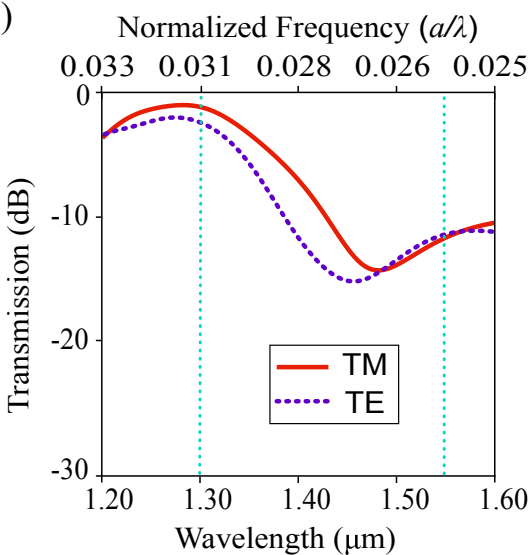

f)

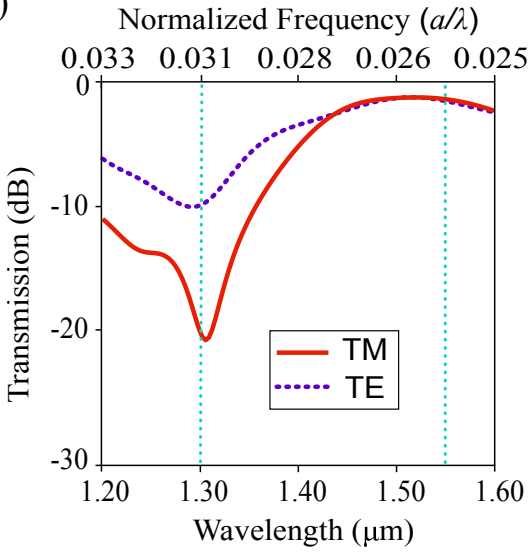

Figure 2. Steady-state (a) magnetic and (b) electric field intensity distributions for TE and TM polarized waves operating at $1.30 \mu \mathrm{m}(\mathrm{a} / \lambda=0.031)$, and $(\mathrm{c})$ transmission efficiencies of the upper waveguide for both TM (solid-line) and TE (dashed-line) polarizations. Similarly, steady-state (d) magnetic and (e) electric field intensity distributions for TE and TM polarized waves operating at $1.55 \mu \mathrm{m}(\mathrm{a} / \lambda=0.0258)$, and (f) transmission efficiencies of the lower waveguide for both TM (solid-line) and TE (dashed-line) polarizations. Vertical dashed lines in (c) and (f) denote the transmission values of the operational wavelengths.

In Fig. 2(a) and 2(b), the input source operating at a wavelength of $1.30 \mu \mathrm{m}(\mathrm{a} / \lambda=0.031)$ shows signs of a strong confinement of light through WG-II. Fig. 2(c) shows the transmission values of the fundamental TE and TM modes for WG-II, which are -1.8 and $-0.99 \mathrm{~dB}$ at $\mathrm{a} / \lambda=0.031$, respectively. A relatively high transmission of over $-2 \mathrm{~dB}$ is obtained within a wide frequency interval of $\mathrm{a} / \lambda=[0.030,0.033]$ for TM polarization. The crosstalk values are $-7.90 \mathrm{~dB}$ for TE and $-8.83 \mathrm{~dB}$ for TM. On the other hand, Fig. 2(d) shows the magnetic field intensity with a clear collimation of the fundamental mode TE for an operating wavelength of $1.55 \mu \mathrm{m}(\mathrm{a} / \lambda=0.0258)$. In addition, for the same channel WG-I, we can see that the electric field intensity in Fig. 2(e) has a slightly sharper rotation through it. Fig. 2(f) shows the transmission values at a/ $\lambda=0.0258$ for WG-I for TE and TM are -1.65 and $-1.48 \mathrm{~dB}$, respectively. The crosstalk values are lesser than the upper channel, i.e., -9.35 $\mathrm{dB}$ for TE and $-19.54 \mathrm{~dB}$ for TM polarization. It is important to note that the simulation was run under high-performance meshing $(\mathrm{a} / 4)$ and calculation conditions. The results show that the most important objective of the inverse design optimization is accomplished, that is, a polarization-insensitive wavelength-division is formed without a significant loss for both TE and TM polarizations. Moreover, the complexity of the structure produced by the algorithm is clear, and there is no doubt that such a structure cannot be designed intuitively. 


\section{Experimental verification of the designed PIWDM structure}

Based on normalized frequency values in Section 2, the design can be applied to the desired spectrum region. Microwave experiments were conducted to demonstrate the operational principle of the PIWDM between normalized frequency intervals of $\mathrm{a} / \lambda=[0.024,0.032]$, as in the numerical analysis. The lattice constant is transformed to $\mathrm{a}=0.80 \mathrm{~mm}$ by scaling the operation wavelengths from $1.30 \mu \mathrm{m}(\mathrm{a} / \lambda=0.031)$ and $1.55 \mu \mathrm{m}(\mathrm{a} / \lambda=0.0258)$ to $11.54 \mathrm{GHz}(\mathrm{a} / \lambda=0.031)$ and $9.68 \mathrm{GHz}(\mathrm{a} / \lambda=0.0258)$, respectively. The designed PIWDM structure is fabricated using a "Creality Ender 3 Pro" 3D printer, which uses the PLA material "ESUN PLA + Silver" as a filament. PLA is a low-cost, biodegradable, and all-dielectric material with a low refractive index of $n_{P L A} \cong 1.55$ at $10 \mathrm{GHz}$. Moreover, a 3D printer is arranged for printing with a $100 \%$ infill ratio to obtain the solid and homogenous distribution of the PLA.

a)

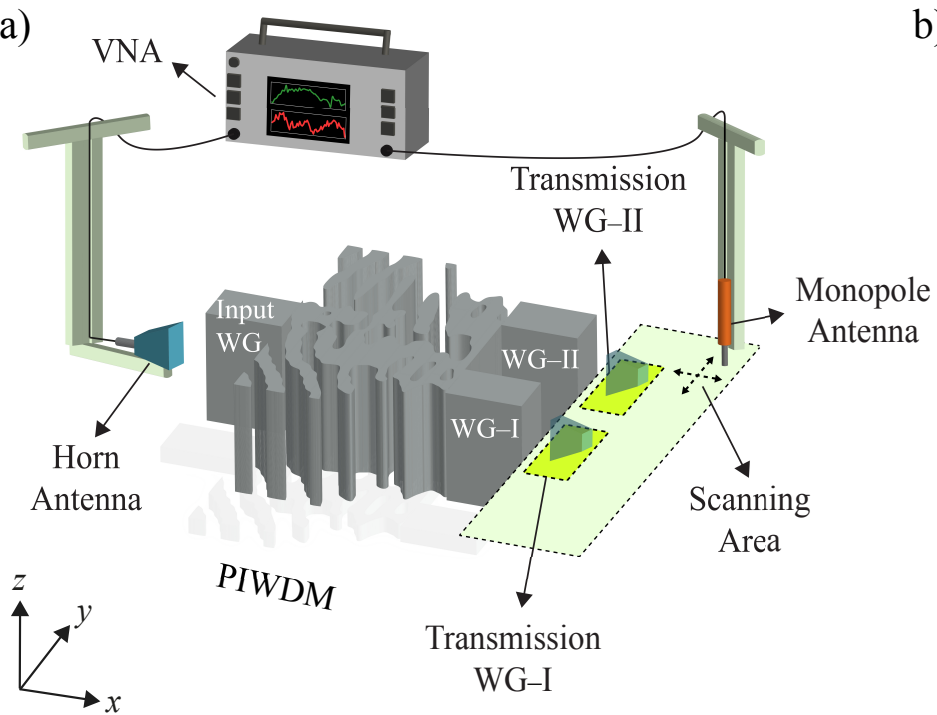

b)

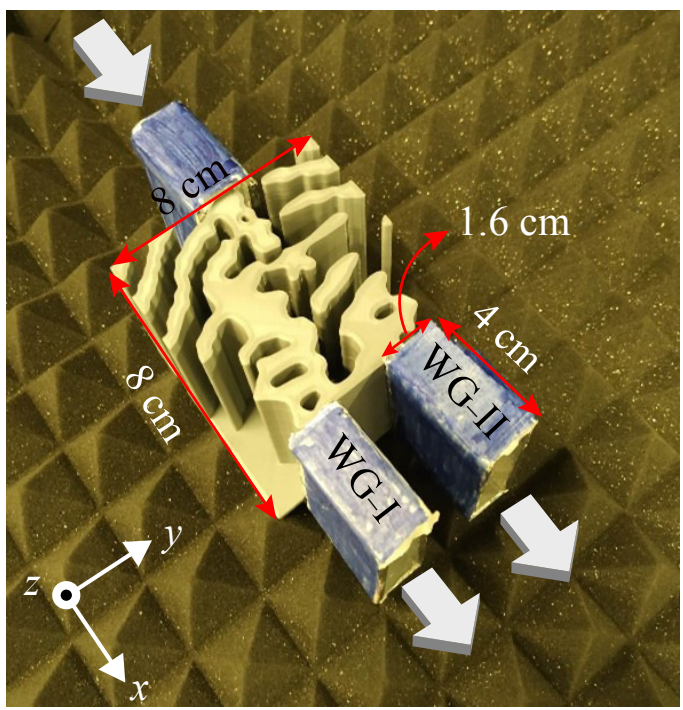

Figure 3. (a) Schematic view of the experimental setup to scan electric and magnetic field intensity distributions (scanning area) and calculate the normalized transmission at the end of the output waveguides (yellow areas). (b) Photographic view of the PIWDM and incorporated waveguides with their structural parameters.

Fig. 3(a) shows a schematic illustration of the experimental setup used to elucidate the wavelength division performance of the PIWDM and its transmission efficiencies. Both horn and monopole antennas are employed to transmit and receive Gaussian profiled electromagnetic waves through Agilent E5071C ENA vector network analyzer (VNA), respectively. During the experiments, the xy-plane (scanning area) was scanned to obtain the electric field $\left|E_{z}\right|^{2}$ and magnetic field $\left|H_{z}\right|^{2}$ intensity distributions by arranging the appropriate orientations of the horn and monopole antennas for both TM and TE polarizations, respectively. In addition, another horn antenna, which is identical to the transmitter antenna, is utilized to measure the transmission values at the end of the output waveguides (WG-I and WG-II) for each polarization. A photographic illustration of the 3D-printed PIWDM and the constructed waveguides along with their physical dimensions are presented in Fig. 3(b). The width and length of the PIWDM emerged as $8 \mathrm{~cm}$, whereas the thickness was $6 \mathrm{~cm}$. Furthermore, input and output waveguides are constructed using hollow rectangular aluminum foil coated cases to couple the light within the microwave regime. Moreover, these cases are considered to eliminate the absorption losses by ensuring that undesired reflections inside the cases are eliminated owing to the flat coating of the foils and guide and detect the wave at the input and output ports. All waveguides are almost identical and have spatial dimensions equal to $4 \mathrm{~cm}$ (length), $1.60 \mathrm{~cm}$ (width), and $6 \mathrm{~cm}$ (height) for the $\mathrm{x}-, \mathrm{y}-$, and $\mathrm{z}$-directions, respectively.

For field intensity measurements, the monopole antenna was moved by 2 -mm steps along both the $\mathrm{x}$ - and $\mathrm{y}$-axes, and a precise interpolation was then applied to obtain the representative results. Similar to the numerical measurements in the simulations, the level of the z-axis is set to the middle of the structure. Fig. 4(a) and 4(b) show the field intensity distributions at $9.68 \mathrm{GHz}(\mathrm{a} / \lambda=0.0258)$ for the TM and TE polarizations, respectively. The longitudinal normalized cross-sectional profiles are superimposed to clearly illustrate the intensity differences at the end of the output waveguides. Similarly, the electric and magnetic field intensity distributions are shown in Fig. 4(d) and 4(e) for TM and TE polarizations at $11.74 \mathrm{GHz}(\mathrm{a} / \lambda=$ 0.0313), respectively, with their normalized cross-sectional profiles. All field distributions clearly illustrate the separation of both 9.68 and $11.74 \mathrm{GHz}$ frequencies at the end of the output waveguides. Two identical horn antennas were used to obtain the transmission spectrums of the PIWDM structure between 9 and $12 \mathrm{GHz}$. One of the horn antennas is used to inject microwaves 
a)

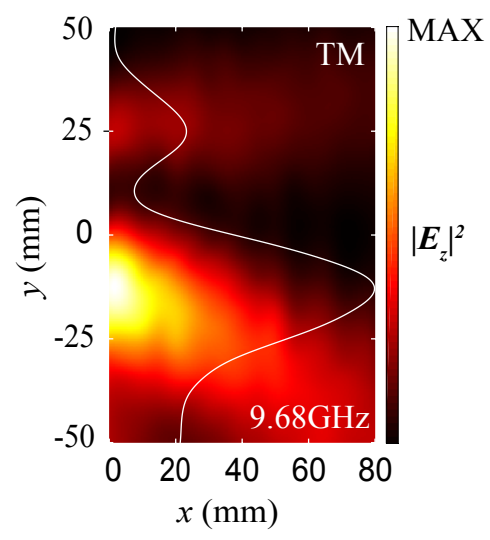

d)

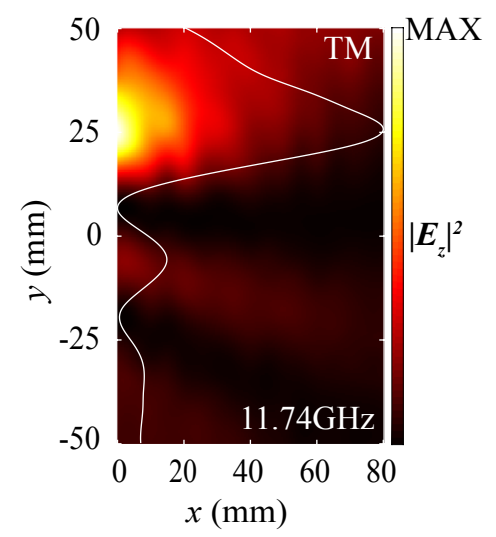

b)

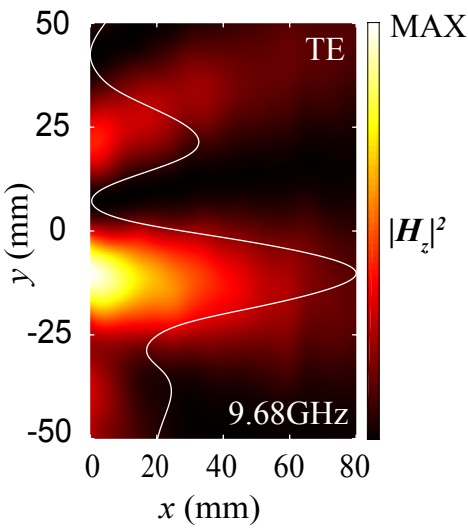

e)

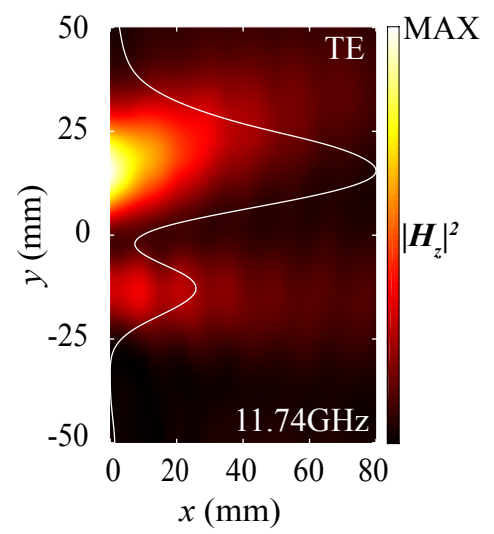

c)

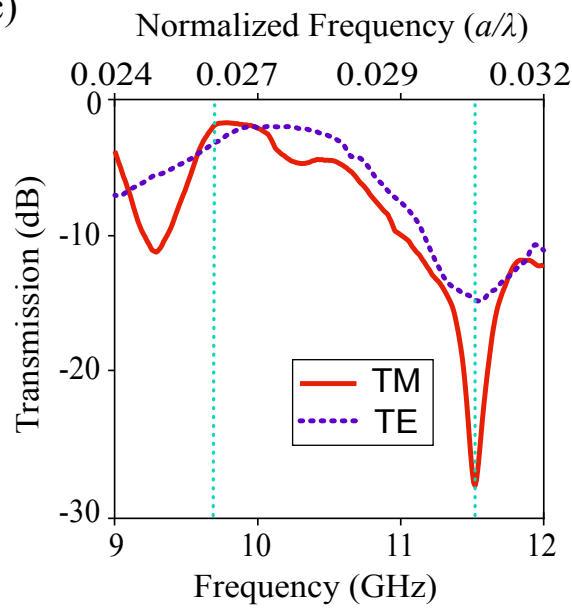

f)

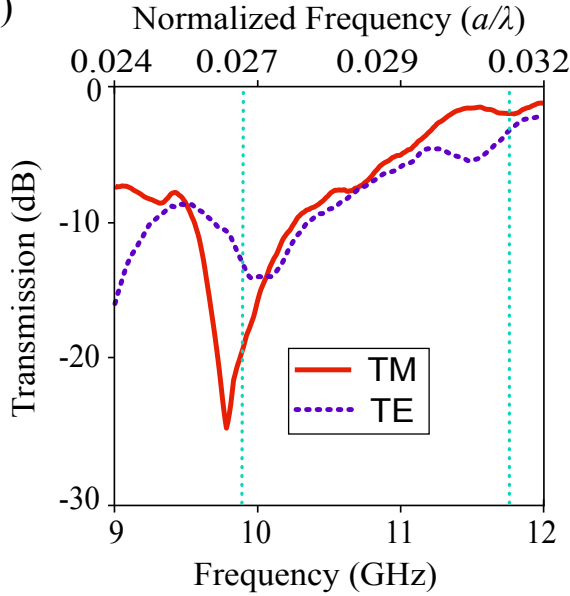

Figure 4. (a) Electric and (b) magnetic field intensities for TM and TE polarizations operating at $9.68 \mathrm{GHz}(\mathrm{a} / \lambda=0.0258)$, and (c) transmission efficiencies of lower waveguide (WG-I) for both TM (solid-line) and TE (dotted-line) polarizations. Similarly, (d) electric and (e) magnetic field intensities for TM and TE polarizations operating at $11.74 \mathrm{GHz}(\mathrm{a} / \lambda=0.0313)$, and (f) transmission efficiencies of upper waveguide (WG-II) for both TM (solid-line) and TE (dotted-line) polarizations. The white lines indicate the longitudinal normalized cross-sectional profiles. Vertical dashed lines in (c) and (f) denote the transmission values of operational frequencies.

as in the previous experiment and the other one is employed to measure the transmission values at the end of the outputs WG-I and WG-II for all frequencies. Free space transmission values were also measured to normalize the data for both output waveguides and polarizations. Fig. 4(c) and 4(f) show the normalized transmission for the output WG-I and output WG-II for both TM (solid line) and TE (dotted line) polarizations, respectively. For WG-I, the transmissions are -2.23 and $-2.18 \mathrm{~dB}$ at $9.68 \mathrm{GHz}$ for TM and TE polarizations, respectively. For TM polarization, the crosstalk is measured as $-12.77 \mathrm{~dB}$, whereas it is $-12.80 \mathrm{~dB}$ for TE polarization. Unlike WG-I, the transmission values measured at the end of the WG-II experienced a slight shift of approximately $0.20 \mathrm{GHz}$ from the exact frequencies. Despite an increase in the transmissions of both polarizations at above $9.88 \mathrm{GHz}$, transmission peaks at $11.74 \mathrm{GHz}$, where the transmission values are -2.19 and $-2.42 \mathrm{~dB}$ for TM and TE polarizations, respectively. In addition, the crosstalk values at $11.74 \mathrm{GHz}$ are calculated as $-18.16 \mathrm{~dB}$ for TM polarization and $-11.45 \mathrm{~dB}$ for TE polarization. Overall, the experimentally measured values of the transmission graphs show the overall functionality of the PIWDM. It is important to note that the possible reasons for the discrepancy between numerical and experimental results include the impurity of the material, the production capability of the 3D printer, and external factors such as temperature and imperfect alignment. In addition, the experiment has a larger coupling loss because of the free space to the dielectric structure coupling loss, owing to the impedance mismatch. The utilized computational algorithm produces a complex and compact metastructure with an ability to arbitrarily control of the light's polarization. The close inspection of the generated dielectric distribution indicates the presence of both connected and isolated material parts as the objective includes the manipulation of both TE and TM polarizations, which have different oscillating planes for the electric and magnetic field components. Besides, 
the continuity of the tangential component of the electric field gives rise to different interactions as the polarization changes. Proposed $1 \times 2$ low index PIWDM design is fabricated by scaling the device from sizes of micrometers to centimeters to realize experimental verifications in microwave region wavelengths. Proof-of-concept of the designed device verified by experimental measurements shows that inverse designed PIWDM can efficiently separate wavelengths into different waveguide channels independently of their polarization state. Final structure exhibits high transmission ratio, low crosstalk values, compactness, and most importantly the independence of polarization, which can bring an important diversity to optical designs. In this regard, besides removing the necessary search for a polarizer, the device could also prove its main function of demultiplexing at 1.30 $\mu \mathrm{m}$ and $1.55 \mu \mathrm{m}$ with great performance for both TE and TM polarizations. Moreover, all listed characteristics of the PIWDM revealed under a low refractive index of 1.55 by which normally the light is expected to have a lower performance because of weak light matter interaction behavior. As a result, the design satisfies the requirements of the deployed multi-objective function with a non-intuitive refractive index configuration.

\section{Conclusion}

In conclusion, we numerically studied a low-index dielectric medium capable of spectral demultiplexing for both TE and TM polarized light at $1.30 \mu \mathrm{m}$ and $1.55 \mu \mathrm{m}$. We used a powerful design tool to independently manipulate TE and TM polarized light. Polarization-controlled low-index devices can find many applications in optical communications and photonic integrated circuits where in-plane photonic devices are essential parts of such applications. PIWDM was designed using a low-index dielectric material (PLA) with the inverse design approach, and the transmission values are between -0.99 and $-1.8 \mathrm{~dB}$. To verify the simulated device, it was produced using 3D printing technology and the experiment was carried out within the microwave region. Due to the low-index nature of the design, light coupling from external laser sources is expected to have low insertion losses as compared to high-index dielectric (such as Silicon) based devices. As a result of the experiment, the transmission values were -2.42 and $-2.18 \mathrm{~dB}$ at WG-II and WG-I waveguides for TE polarization, respectively. However, the transmission values were -2.19 and $-2.23 \mathrm{~dB}$ at WG-II and WG-I waveguides for TM polarization, respectively. Consequently, the experimental results support the design purpose of polarization-independent wavelength separation behavior. These results can pave the way for the inverse design of compact, high-efficiency devices that are polarization-independent, with multiple output ports. With additional purposes added to the design algorithm, the structure can be achieved even with higher efficiency, a larger number of output ports, and even a narrower channel spacing. With the development of production techniques for asymmetric structures, it is thought that such devices can be easily used in commercial products. This might be a breakthrough for optical communications schemes that are widely dependent on polarization control. The PIWDM design is endowing a huge flexibility to the performance improvements of integrated photonic circuits, which may lead the way to several different designs that have more compact area constraints.

\section{References}

1. Kirchain, R. \& Kimerling, L. A roadmap for nanophotonics. Nat. Photonics 1, 303-305, DOI: 10.1038/nphoton.2007.84 (2007).

2. Su, Y., Tian, Y., Wong, E., Nadarajah, N. \& Chan, C. All-optical virtual private network in passive optical networks. Laser \& Photonics Rev. 2, 460-479, DOI: 10.1002/lpor.200810021 (2008).

3. Luo, L.-W. et al. Wdm-compatible mode-division multiplexing on a silicon chip. Nat. Commun. 5, DOI: 10.1038/ ncomms4069 (2014).

4. Senior, J. M. \& Jamro, M. Y. Optical Fiber Communications: Principles and Practice (Financial Times/Prentice Hall, 2009).

5. Chiles, J., Buckley, S. M., Nam, S. W., Mirin, R. P. \& Shainline, J. M. Design, fabrication, and metrology of 10 x 100 multi-planar integrated photonic routing manifolds for neural networks. APL Photonics 3, 106101, DOI: 10.1063/1.5039641 (2018).

6. Hayran, Z. et al. Numerical and experimental demonstration of a wavelength demultiplexer design by point-defect cavity coupled to a tapered photonic crystal waveguide. Opt. Lett. 41, 119, DOI: 10.1364/ol.41.000119 (2015).

7. Jia, H. et al. Wdm-compatible multimode optical switching system-on-chip. Nanophotonics 8, 889-898, DOI: 10.1515/ nanoph-2019-0005 (2019).

8. Dong, P. Silicon photonic integrated circuits for wavelength-division multiplexing applications. IEEE J. Sel. Top. Quantum Electron. 22, 370-378, DOI: 10.1109/jstqe.2016.2575358 (2016).

9. Dai, D. \& Bowers, J. E. Silicon-based on-chip multiplexing technologies and devices for peta-bit optical interconnects. Nanophotonics 3, 283-311, DOI: 10.1515/nanoph-2013-0021 (2014). 
10. Mikkelsen, J. C. et al. Polarization-insensitive silicon nitride mach-zehnder lattice wavelength demultiplexers for cwdm in the o-band. Opt. Express 26, 30076, DOI: 10.1364/oe.26.030076 (2018).

11. Piggott, A. Y. et al. Inverse design and demonstration of a compact and broadband on-chip wavelength demultiplexer. Nat. Photonics 9, 374-377, DOI: 10.1038/nphoton.2015.69 (2015).

12. Yilmaz, Y. A., Alpkilic, A. M., Yeltik, A. \& Kurt, H. Inverse design of efficient and compact 1 x n wavelength demultiplexer. Opt. Commun. 454, 124522, DOI: 10.1016/j.optcom.2019.124522 (2020).

13. Su, L., Piggott, A. Y., Sapra, N. V., Petykiewicz, J. \& Vuckovic, J. Inverse design and demonstration of a compact on-chip narrowband three-channel wavelength demultiplexer. ACS Photonics 5, 301-305, DOI: 10.1021/acsphotonics.7b00987 (2017).

14. Huang, J. et al. Implementation of on-chip multi-channel focusing wavelength demultiplexer with regularized digital metamaterials. Nanophotonics 9, 159-166, DOI: 10.1515/nanoph-2019-0368 (2019).

15. Dangel, R. et al. Development of versatile polymer waveguide flex technology for use in optical interconnects. J. Light. Technol. 31, 3915-3926, DOI: 10.1109/j1t.2013.2282499 (2013).

16. Missinne, J. et al. Stretchable optical waveguides. Opt. Express 22, 4168, DOI: 10.1364/oe.22.004168 (2014).

17. Zuo, H., Yu, S., Gu, T. \& Hu, J. Low loss, flexible single-mode polymer photonics. Opt. Express 27, 11152, DOI: 10.1364/oe.27.011152 (2019).

18. Yu, S. et al. Compact and fabrication-tolerant waveguide bends based on quadratic reflectors. J. Light. Technol. 38, 4368-4373, DOI: 10.1109/jlt.2020.2986576 (2020).

19. Meem, M. et al. Large-area, high-numerical-aperture multi-level diffractive lens via inverse design. Optica 7, 252, DOI: 10.1364/optica.388697 (2020).

20. Jiang, J. \& Fan, J. A. Global optimization of dielectric metasurfaces using a physics-driven neural network. Nano Lett. 19, 5366-5372, DOI: 10.1021/acs.nanolett.9b01857 (2019).

21. So, S., Badloe, T., Noh, J., Bravo-Abad, J. \& Rho, J. Deep learning enabled inverse design in nanophotonics. Nanophotonics 9, 1041-1057, DOI: 10.1515/nanoph-2019-0474 (2020).

22. Jia, H., Zhou, T., Fu, X., Ding, J. \& Yang, L. Inverse-design and demonstration of ultracompact silicon meta-structure mode exchange device. ACS Photonics 5, 1833-1838, DOI: 10.1021/acsphotonics.8b00013 (2018).

23. Yao, K., Unni, R. \& Zheng, Y. Intelligent nanophotonics: merging photonics and artificial intelligence at the nanoscale. Nanophotonics 8, 339-366, DOI: 10.1515/nanoph-2018-0183 (2019).

24. Su, L. et al. Nanophotonic inverse design with spins: Software architecture and practical considerations. Appl. Phys. Rev. 7, 011407, DOI: 10.1063/1.5131263 (2020).

25. Long, Y., Ren, J., Li, Y. \& Chen, H. Inverse design of photonic topological state via machine learning. Appl. Phys. Lett. 114, 181105, DOI: 10.1063/1.5094838 (2019).

26. Forestiere, C., He, Y., Wang, R., Kirby, R. M. \& Negro, L. D. Inverse design of metal nanoparticles' morphology. ACS Photonics 3, 68-78, DOI: 10.1021/acsphotonics.5b00463 (2015).

27. Pestourie, R. et al. Inverse design of large-area metasurfaces. Opt. Express 26, 33732, DOI: 10.1364/oe.26.033732 (2018).

28. Callewaert, F., Butun, S., Li, Z. \& Aydin, K. Inverse design of an ultra-compact broadband optical diode based on asymmetric spatial mode conversion. Sci. Reports 6, DOI: 10.1038/srep32577 (2016).

29. Callewaert, F. et al. Inverse-designed stretchable metalens with tunable focal distance. Appl. Phys. Lett. 112, 091102, DOI: 10.1063/1.5017719 (2018).

30. Elesin, Y., Lazarov, B., Jensen, J. \& Sigmund, O. Time domain topology optimization of 3d nanophotonic devices. Photonics Nanostructures - Fundamentals Appl. 12, 23-33, DOI: 10.1016/j.photonics.2013.07.008 (2014).

31. Sitawarin, C., Jin, W., Lin, Z. \& Rodriguez, A. W. Inverse-designed photonic fibers and metasurfaces for nonlinear frequency conversion [invited]. Photonics Res. 6, DOI: 10.1364/prj.6.000b82 (2018).

32. Nanophotonic fdtd simulation software - lumerical fdtd (2019).

33. Berenger, J.-P. A perfectly matched layer for the absorption of electromagnetic waves. J. Comput. Phys. 114, 185-200, DOI: 10.1006/jcph.1994.1159 (1994).

34. Weir, W. Automatic measurement of complex dielectric constant and permeability at microwave frequencies. Proc. IEEE 62, 33-36, DOI: 10.1109/proc.1974.9382 (1974). 


\section{Acknowledgements}

Authors acknowledge that this research is supported by Roketsan Missiles Inc. and Scientific and Technological Research Council of Turkey under Award No: 116F200. Authors also would like to thank Bugra Melemen for his contributions in prototyping the experimental device. H.Kurt acknowledges partial support from the Turkish Academy of Sciences.

\section{Author contributions}

A.B.I., A.M.A., and Y.A.Y. designed, A.M.A., Y.A.Y., and B.K.Y. performed the experiments; M.T. and H.K. analyzed the data; A.M.A. and B.K.Y. prepared the figures; and A.B.I., A.M.A., Y.A.Y., B.K.Y., M.T., and H.K. all revised/wrote the paper.

\section{Funding}

Open Access funding provided by Roketsan Missiles Inc.

\section{Competing interests}

The authors declare no competing interests.

\section{Additional information}

Correspondance and requests for materials should be addressed to A.B.I.

Reprints and permissions information is available at www.nature.com/reprints.

Publisher's note Springer Nature remains neutral with regard to jurisdictional claims in published maps and institutional affiliations. 


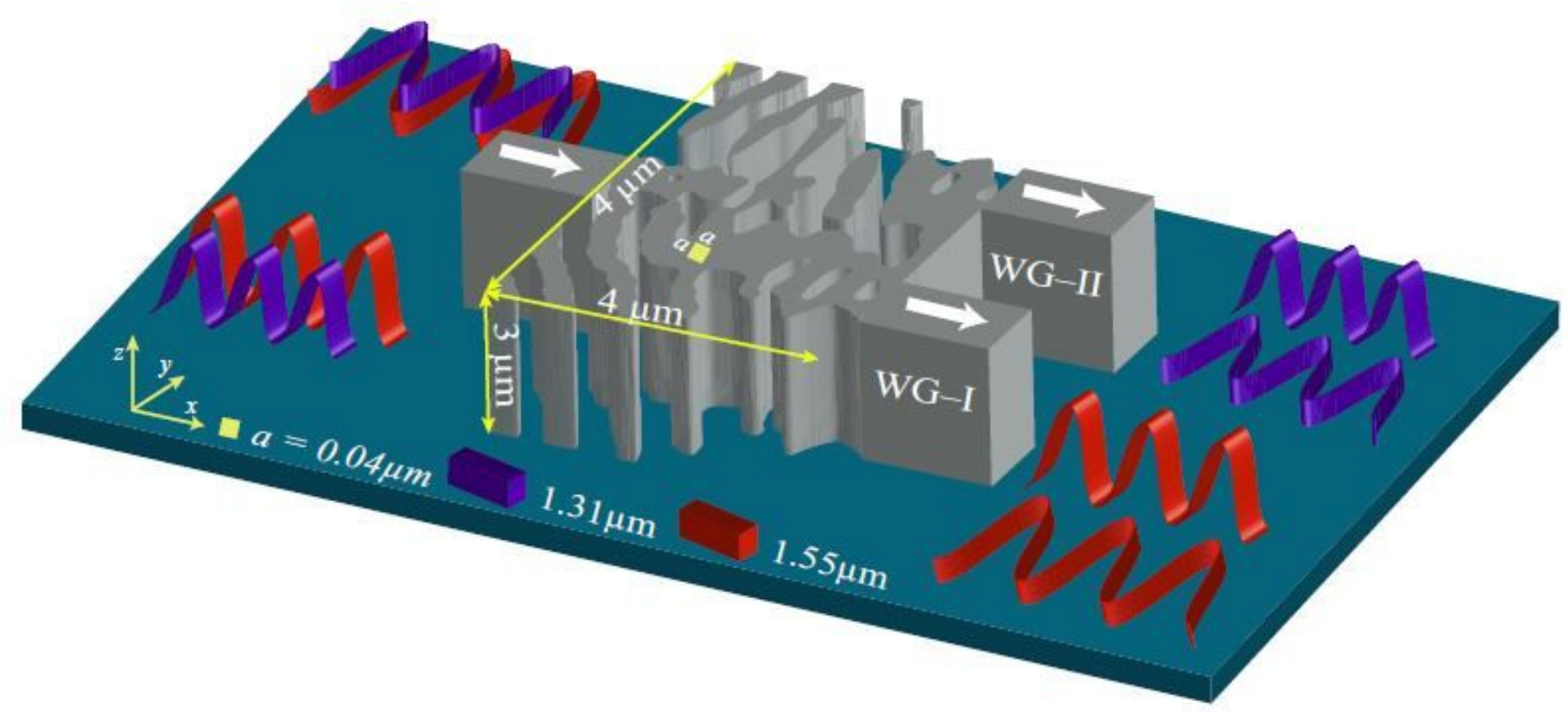

\section{Figure 1}

Schematic depiction of the PIWDM device. Incident light with two different polarizations (vertical and horizontal) oscillating at two specific wavelengths (two colors) is separated irrespective of its polarization. 
a)

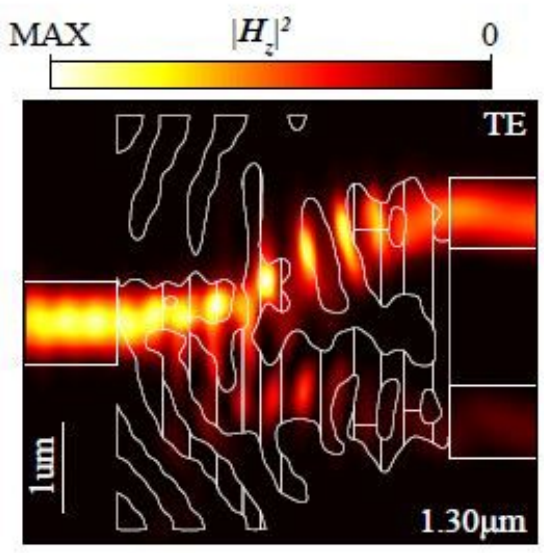

d)

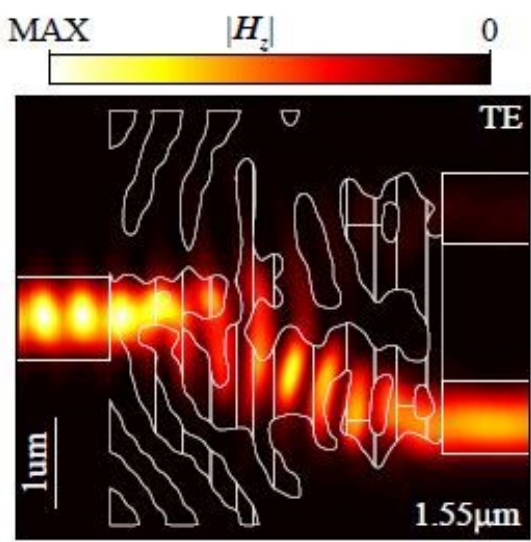

b)

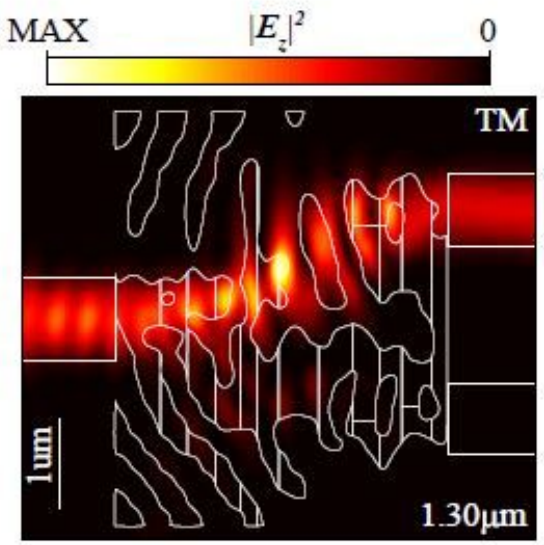

e)

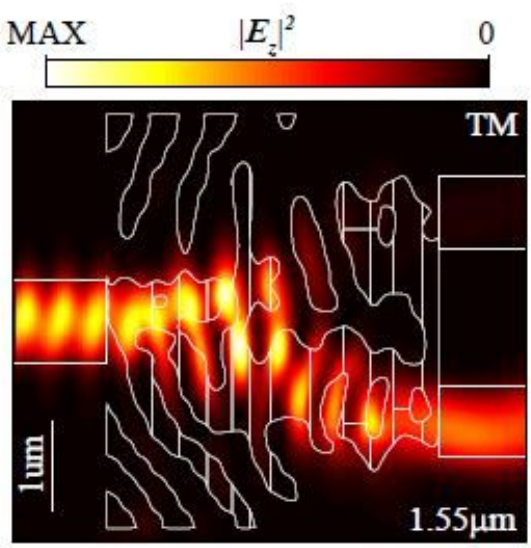

c)

Normalized Frequency $(a / \lambda)$

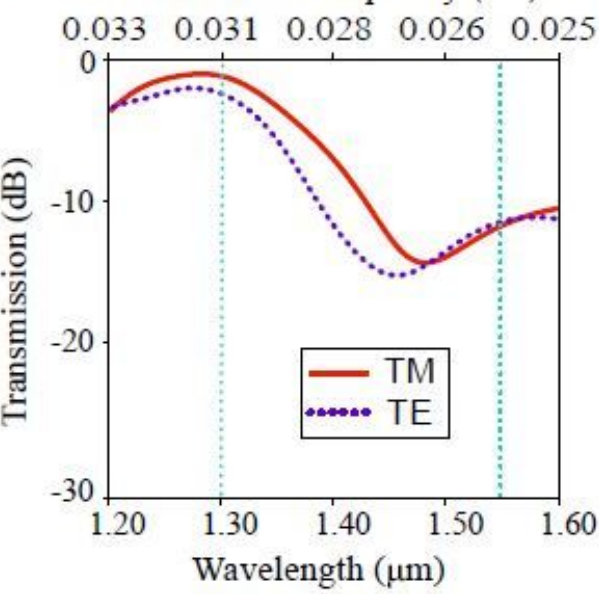

f)

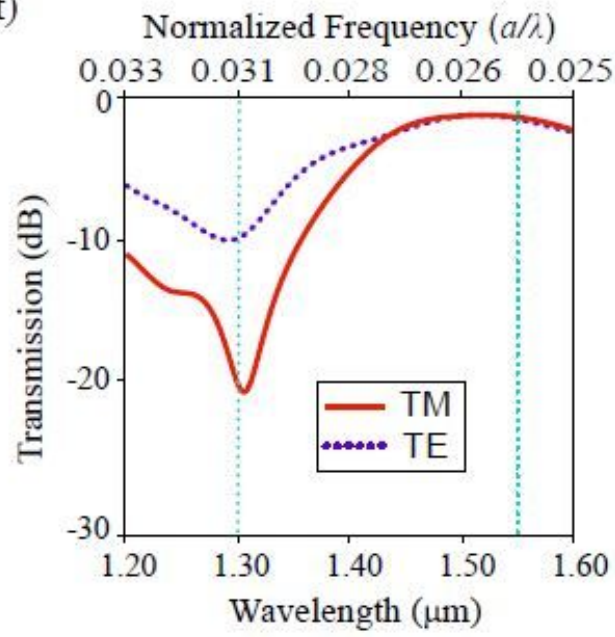

\section{Figure 2}

Steady-state (a) magnetic and (b) electric field intensity distributions for TE and TM polarized waves operating at $1.30 \mathrm{~mm}(\mathrm{a} / \mathrm{I}=0.031)$, and $(\mathrm{c})$ transmission efficiencies of the upper waveguide for both TM (solid-line) and TE (dashed-line) polarizations. Similarly, steady-state (d) magnetic and (e) electric field intensity distributions for TE and TM polarized waves operating at $1.55 \mathrm{~mm}(\mathrm{a} / \mathrm{I}=0.0258)$, and $(\mathrm{f})$ transmission efficiencies of the lower waveguide for both TM (solid-line) and TE (dashed-line) polarizations. Vertical dashed lines in (c) and (f) denote the transmission values of the operational wavelengths. 

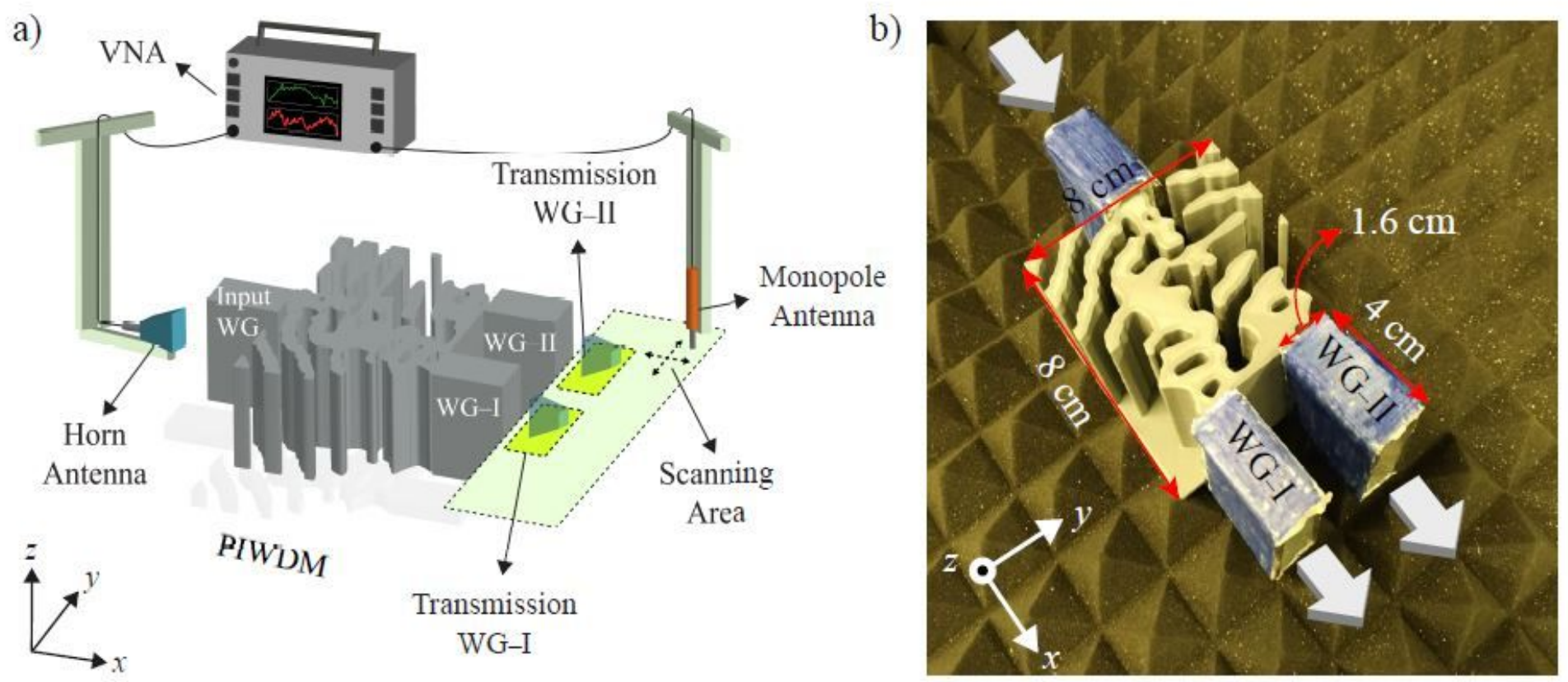

\section{Figure 3}

(a) Schematic view of the experimental setup to scan electric and magnetic field intensity distributions (scanning area) and calculate the normalized transmission at the end of the output waveguides (yellow areas). (b) Photographic view of the PIWDM and incorporated waveguides with their structural parameters. 
a)

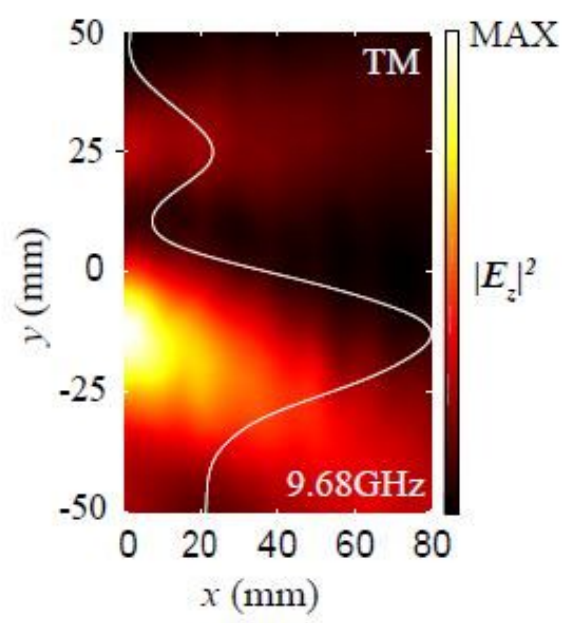

d)

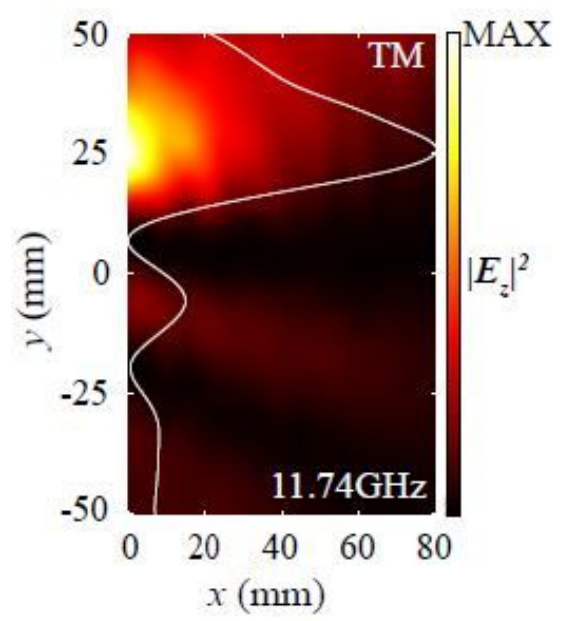

b)

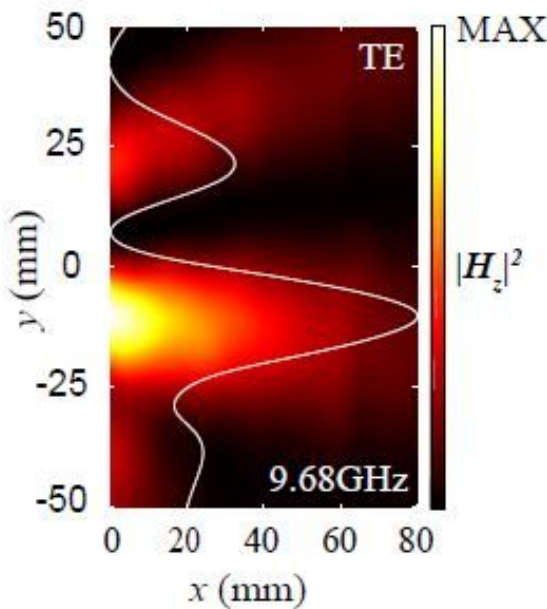

e)

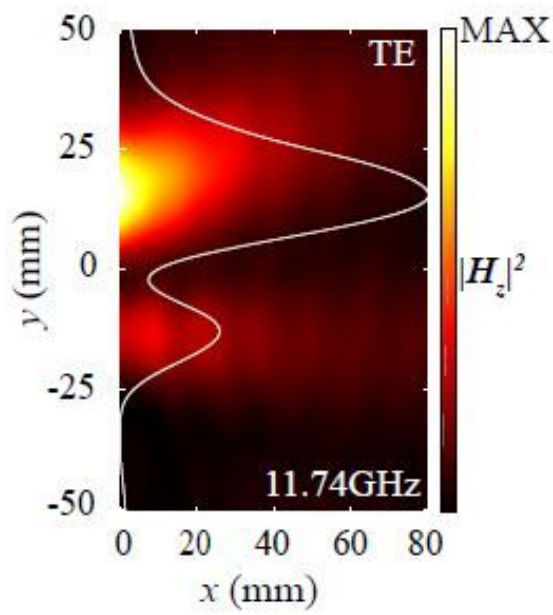

c) Normalized Frequency $(a / \lambda)$

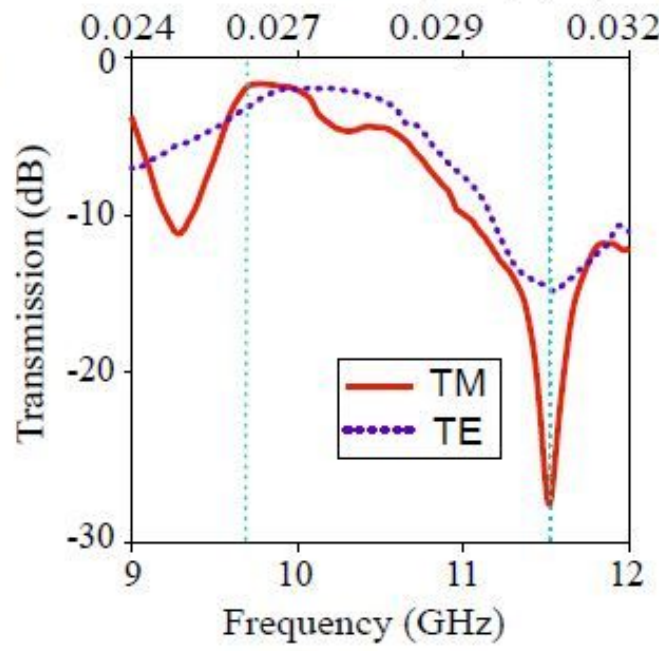

f)

Normalized Frequency $(a / \lambda)$

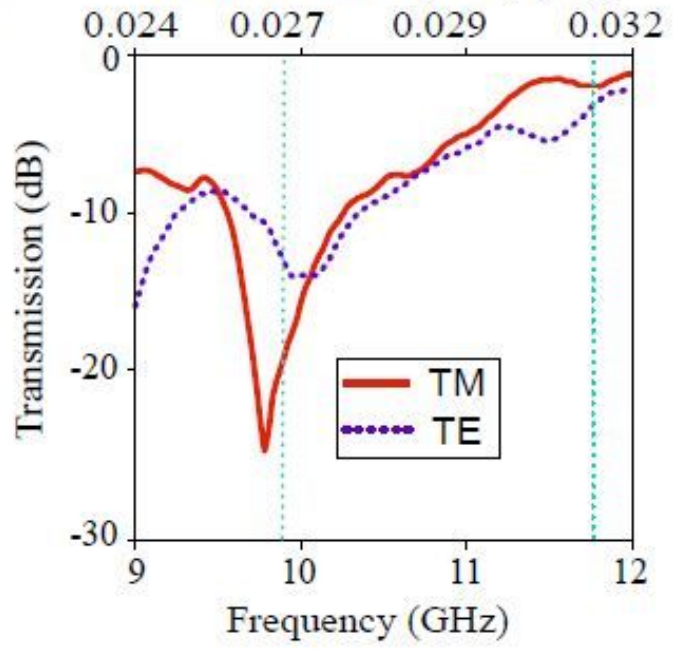

Figure 4

(a) Electric and (b) magnetic field intensities for TM and TE polarizations operating at $9.68 \mathrm{GHz}(\mathrm{a} / \mathrm{I}=$ 0.0258), and (c) transmission efficiencies of lower waveguide (WG-I) for both TM (solid-line) and TE (dotted-line) polarizations. Similarly, (d) electric and (e) magnetic field intensities for TM and TE polarizations operating at $11.74 \mathrm{GHz}(\mathrm{a} / \mathrm{I}=0.0313)$, and $(\mathrm{f})$ transmission efficiencies of upper waveguide (WG-II) for both TM (solid-line) and TE (dotted-line) polarizations. The white lines indicate the longitudinal normalized cross-sectional profiles. Vertical dashed lines in (c) and (f) denote the transmission values of operational frequencies. 